\title{
Importance of collisions with charged particles for stellar UV line shapes: Cd III ${ }^{\star}$
}

\author{
N. Milovanović1,2, M. S. Dimitrijević ${ }^{1,2}$, L. Č. Popović ${ }^{1,2}$, and Z. Simić ${ }^{1,2}$ \\ 1 Astronomical Observatory, Volgina 7, 11160 Belgrade, Serbia and Montenegro \\ 2 Isaac Newton Institute of Chile, Yugoslavia Branch
}

Received 6 August 2003 / Accepted 10 November 2003

\begin{abstract}
Stark broadening parameters, widths and shifts, for 84 spectral lines of doubly-ionized cadmium (Cd III) have been calculated using the modified semi-empirical approach (MSE). Calculated spectral lines belong to the transitions $4 \mathrm{~d}^{9} 5 \mathrm{~s}-4 \mathrm{~d}^{9} 5 \mathrm{p}$ and $4 d^{9} 5 p-4 d^{9} 5 d$. Widths and shifts of the spectral lines are given for temperature range of $5000 \mathrm{~K}-60000 \mathrm{~K}$ and an electron density of $10^{23} \mathrm{~m}^{-3}$. The influence of collisions with charged particles on Cd III UV stellar lines along the HR diagram is discussed. Compared to the Doppler broadening, the influence of the Stark broadening mechanism is more important for deeper atmospheric layers and for larger values of $\log g$. Stark broadening does not depend on turbulent velocity for the considered stellar model of a standard main sequence hot star of A0 spectral type. The influence of the Stark widths for standard models of DA and DB white dwarfs is also discussed.
\end{abstract}

Key words. atomic processes - line: formation - stars: atmospheres - stars: early-type - stars: white dwarfs

\section{Introduction}

Spectral lines of multiply charged heavy elements are present in the UV spectra of early-type stars, especially in spectra of chemically peculiar (CP) ones. Investigation of these lines is important for example for spectral lines synthesis, diagnostics and modelling of laboratory and stellar plasma, abundance determination and opacity calculation.

Stark broadening of spectral lines is the dominant pressure broadening mechanism in hot, early-type, stars and white dwarf atmospheres. This type of broadening might also be important in interstellar molecular and ionized hydrogen clouds, as e.g. for radio recombination spectral lines in W51 emission nebulae (Lang \& Wilson 1978), and in cooler stars such as solar type ones for transitions involving higher principal quantum numbers (Vince \& Dimitrijević 1985).

Spectral lines of $\mathrm{CdI}$ and $\mathrm{Cd}$ II are observed in stellar spectra of some CP stars, e.g. $\chi$ Lupi (Leckrone et al. 1999), so cadmium in various ionization stages is present in stellar atmospheres. With the development of space-born telescopes, spectral instruments like the Goddard High Resolution Spectrograph (GHRS) on the Hubble Space Telescope provide good resolution spectra of stellar objects so that the need for trace element data, like that for cadmium, increases.

Send offprint requests to: N. Milovanović,

e-mail: nmilovanovic@aob.bg.ac.yu

* Tables 1 and 2 are only available in electronic form at the CDS via anonymous ftp to cdsarc.u-strasbg. fr $(130.79 .128 .5)$ or via http://cdsweb.u-strasbg.fr/cgi-bin/qcat?]/A+A/417/375
Here, we present the computed electron-impact broadening parameters, widths and shifts, for 84 spectral lines (12 singlets and 72 triplets) of Cd III obtained using the modified semiempirical method - MSE (Dimitrijević \& Konjević 1980; Dimitrijević \& Kršljanin 1986).

\section{Method of calculation}

Advanced calculation of the Stark broadening parameters using the strong-coupling quantum-mechanical method (Baranger 1958a, 1958b, 1958c; Kolb \& Griem 1958; Griem 1974; Dimitrijević et al. 1981; Seaton 1988; Ralchenko et al. 1999; Zeng-xin et al. 1999) is so complicated that only a limited amount of data for spectral lines originating from lowlying transitions can be calculated adequately. On the other hand, the semi-classical method (Sahal-Bréchot 1969a, 1969b; Dimitrijević et al. 1991; see also a review of obtained results in Dimitrijević 1997) needs a large amount of atomic data, energy levels and oscillator strengths. This method is not applicable in an adequate way to the Stark broadening calculation of Cd III because there is insufficient number of reliable atomic data.

Here we use the modified semi-empirical approach (MSE, Dimitrijević \& Konjević 1980; Dimitrijević \& Kršljanin 1986) which includes explicitly only levels with $\Delta n=0$ and $l_{\text {if }}^{\prime}=$ $l_{\text {if }} \pm 1$, where $n$ is the principal quantum number, $l$ is the orbital quantum number and $i$ and $f$ denote initial and final level, respectively. Levels with $\Delta n \neq 0$ are combined and approximately estimated, so that for Stark broadening parameter calculations we need less atomic data then in the semi-classical 
method. We note that the needed dipole matrix elements have been calculated using the Coulomb approximation method described in Bates \& Damgaard (1949), Shore \& Menzel (1965) and Oertel \& Shomo (1967).

The modified semi-empirical method is applicable under the following conditions:

1. That the line is isolated, i.e. non-degenerate energy levels broadened by collisions do not overlap (Sahal-Bréchot 1969a; Dimitrijević \& Sahal-Bréchot 1984).

2. That the minimal distance of the perturbing electron from an emitting particle is significantly smaller than distances between two perturbing electrons, i.e. that the probability that two or more electrons are close to an emitting particle is small (see e.g. Griem 1974).

3. That we can assume an impact approximation, which requires that the collision volume $V \sim \rho^{3}$ multiplied by electron density is much less than 1 (Sahal-Bréchot 1969a).

4. That the plasma is ideal. With the increase of the density the number of particles in the Debye sphere decreases and the plasma becomes non-ideal (Konjević \& Uzelac 1990). The idealness of the plasma can be checked by calculating the number of perturbers in the Debye sphere which must be larger than 1 (Dimitrijević et al. 1991).

The accuracy of the MSE calculations for spectral line widths is around $\pm 50 \%$ (Dimitrijević \& Konjević 1980). The average ratio of calculated and experimental widths, determined in Dimitrijević \& Konjević (1980) for 36 multiplets of 7 different doubly-charged ions is $1.06 \pm 0.32$ and for 7 multiplets for 4 triply-charged ions $0.91 \pm 0.42$. For 71 multiplets of 7 singlycharged ions with various couplings schemes Popović (1994) found that the overall average ratio of experimental to calculated widths is $1.4 \pm 0.3$. Even for relatively complex spectra of Xe II and Kr II with mixed jK and LS couplings, the accuracy found is about $\pm 30 \%$ (Popović \& Dimitrijević 1996). The accuracy of shifts usually gives higher errors compared to experimental values. This is a consequence of the cancellations of important contributions with different signs (see Dimitrijević \& Kršljanin 1986). The error of obtained shifts with MSE calculations is within $\pm 50 \%$ of the corresponding width value.

\section{Results}

Doubly-ionized cadmium (Cd III) belongs to the palladium isoelectronic sequence with the ground state electronic configuration $4 \mathrm{~d}^{10}{ }^{1} S_{0}$ and ionization potential of $302200 \pm 50 \mathrm{~cm}^{-1}$. Atomic data needed for our MSE calculation were taken from Van Kleef et al. (1980). They observed Cd III spectra in the UV spectral range from 50 to $210 \mathrm{~nm}$ with the 6.65 and $10.7 \mathrm{~m}$ normal-incidence vacuum spectrograph. Experimental values of energy levels were checked with the least-square level fitting of Hartee-Fock (HF) calculations of atomic parameters. They give a more complete analysis of energy level values compared to the first observed spectra of Cd III presented in the paper of Shenstone \& Pittenger (1949). Van Kleef et al. (1980) estimated that the energy levels error for sharp lines is $\pm 0.5 \mathrm{~cm}^{-3}$ and several $\mathrm{cm}^{-3}$ for strong and asymmetric lines.
Even if coupling schemes slowly go from LS to jj coupling as we go through the isoelectronic sequence from $\mathrm{Pd} I$ to Sn V, most of the energy levels, without losing accuracy, can be represented with LS coupling (van Kleef et al. 1980). Consequently an LS coupling scheme is adopted here.

The calculated Stark widths and shifts of Cd III spectral lines are given in Table 1 (available only in electronic form) as a function of temperature (from 5000 to $60000 \mathrm{~K}$ ) for an electron density of $N=10^{23} \mathrm{~m}^{-3}$. In Table 1 we present results for 84 spectral lines, where 22 belong to $4 d^{9} 5 s-4 d^{9} 5 p$ and 62 to $4 \mathrm{~d}^{9} 5 \mathrm{p}-4 \mathrm{~d}^{9} 5 \mathrm{~d}$ transition.

For spectrum synthesis programs, as e.g. the frequently used SYNTH code (Piskunov 1992), it is more convenient to present the temperature dependence of Stark width by using parameters $A_{0}, A_{1}$ and Stark widths $w\left[\mathrm{rad} \mathrm{s}^{-1}\right]$ per electron for temperature $T=10000 \mathrm{~K}$. These parameters can be derived numerically from the best fit of data given in Table 1 using equation (see Popović et al. 1999)

$\frac{w\left[\mathrm{rad} \mathrm{s}^{-1}\right]}{N}=A_{0} \cdot T^{A_{1}}$,

where

$w\left[\mathrm{rad} \mathrm{s}^{-1}\right]=w[\mathrm{~nm}] \frac{2 \pi c \times 10^{-8}}{\lambda^{2}[\mathrm{~nm}]}$.

The parameters $\log A_{0}, A_{1}$ and $\log w\left[\mathrm{rad} \mathrm{s}^{-1}\right]$ for 84 spectral lines are given in Table 2 (available only in electronic form).

The Eq. (1) may be used for reasonable extrapolations toward higher temperatures. It is not advisable to extrapolate toward temperatures lower than given in Table $1(T<5000 \mathrm{~K})$, without checking the validity of the impact approximation. Moreover, at lower temperatures, the importance of quantum effects increases and the application of MSE method as well as the other semi-classical, classical or semi-empirical ones neglecting quantum effects becomes questionable.

The extrapolation toward lower electron densities $N$ is linear. For higher densities however, it is preferable to check the influence of Debye shielding, non-ideal plasma effect and that the spectral line is isolated.

\section{Discussion}

We used our results for Stark widths given in Tables 1 and 2 to examine the importance of electron-impact broadening in hot stars and white dwarf atmospheres for trace elements like Cd III. Behavior of Stark and Doppler spectral line widths in stellar atmospheres were calculated for Cd III $5 p{ }^{3} \mathrm{~F}_{3}^{0}-5 \mathrm{~d}^{3} \mathrm{G}_{3}$ $(\lambda=144.754 \mathrm{~nm})$, a strong spectral line in various atmospheric models.

These calculations were performed for solar element abundance atmospheric models given in Kurucz (1979) and Kurucz's web site (http://kurucz.harvard.edu/). Each model is characterized by the effective temperature $T_{\text {eff }}$, logarithm of gravity $\log g$ and turbulent velocity $v_{\mathrm{t}}$ and each atmospheric layer within the model is characterized by electron density $N$ and temperature $T$. Stark widths for $\lambda=144.754 \mathrm{~nm}$ spectral line for a particular model were calculated using Eq. (1). In this equation the Stark width is a function of $N$ 


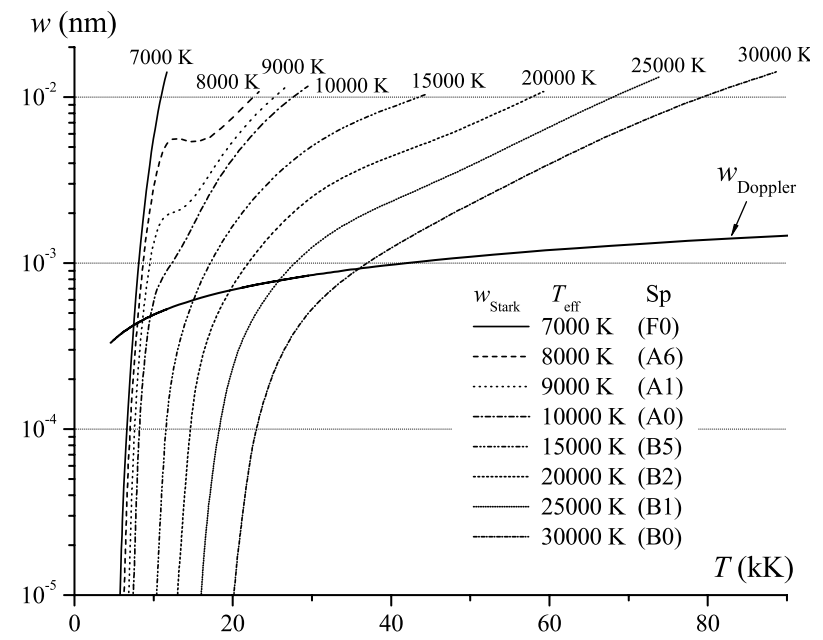

Fig. 1. Stark widths (FWHM) (thinner lines) and Doppler width (thicker line) for Cd III $5 \mathrm{p}{ }^{3} \mathrm{~F}_{3}^{0}-5 \mathrm{~d}^{3} \mathrm{G}_{3}(\lambda=144.754 \mathrm{~nm})$ spectral line as a function of atmospheric layer temperatures. Stark widths are shown for 8 atmospheric models with effective temperatures $T_{\text {eff }}=$ 7000-30000 K, corresponding to spectral classes (Sp) from F0 to B0, $\log g=4.0$ and turbulent velocity $v_{\mathrm{t}}=0 \mathrm{~km} \mathrm{~s}^{-1}$.

and $T$. For these line parameters $\log A_{0}$ is -3.64248 and $A_{1}=$ -0.50410 .

In hot star atmospheres, besides electron-impact broadening (Stark broadening) the important broadening mechanism is a Doppler (thermal) one as well as the broadening due to the turbulence and stellar rotation. Other types of spectral line broadening, such as van der Waals, resonance and natural broadening, are usually negligible. For a Doppler-broadened spectral line the intensity distribution is not Lorentzian as for electron-impact broadening but Gaussian, whose full half width of the spectral line may be determined by the equation (see e.g. Konjević 1999)

$w_{\mathrm{D}}[\mathrm{nm}]=7.16 \times 10^{-7} \lambda[\mathrm{nm}] \sqrt{\frac{T[\mathrm{~K}]}{M_{\mathrm{Cd}}}}$,

where atomic weight for cadmium is $M_{\mathrm{Cd}}=112.411$ a.u.

The importance of Stark broadening in hot star atmospheres is illustrated in Figs. 1-5. In Fig. 1 Stark (FWHM) and Doppler widths for Cd III $5 p{ }^{3} \mathrm{~F}_{3}^{0}-5 \mathrm{~d}^{3} \mathrm{G}_{3}(\lambda=144.754 \mathrm{~nm})$ spectral line as a function of atmospheric layer temperatures are shown. Stark widths are shown for 8 atmospheric models with effective temperatures $T_{\text {eff }}=7000-30000 \mathrm{~K}$, corresponding to spectral classes (Sp) from F0 to B0, logarithm of surface gravity $\log g=4.0$ and turbulent velocity $v_{\mathrm{t}}=0 \mathrm{~km} \mathrm{~s}^{-1}$. In Fig. 1 one can see that Stark widths are lager than Doppler ones for stars with lower effective temperatures. For stars with higher effective temperatures, Stark broadening is more important than Doppler one for deeper atmospheric layers (larger layer temperature $T$ ). For example, for stars with effective temperature $T_{\text {eff }}=30000 \mathrm{~K}$ (B0 stars), Stark and Doppler widths are equal for temperature layer $T \approx 35000 \mathrm{~K}$ and for stars with $T_{\text {eff }}=7000 \mathrm{~K}$ (F0 stars), they are equal for $T \approx 5000 \mathrm{~K}$.

The dependence of Stark and Doppler broadening on atmospheric layer temperature for 7 values of surface gravity is shown in Fig. 2. Models used here have $T_{\text {eff }}=10000 \mathrm{~K}$

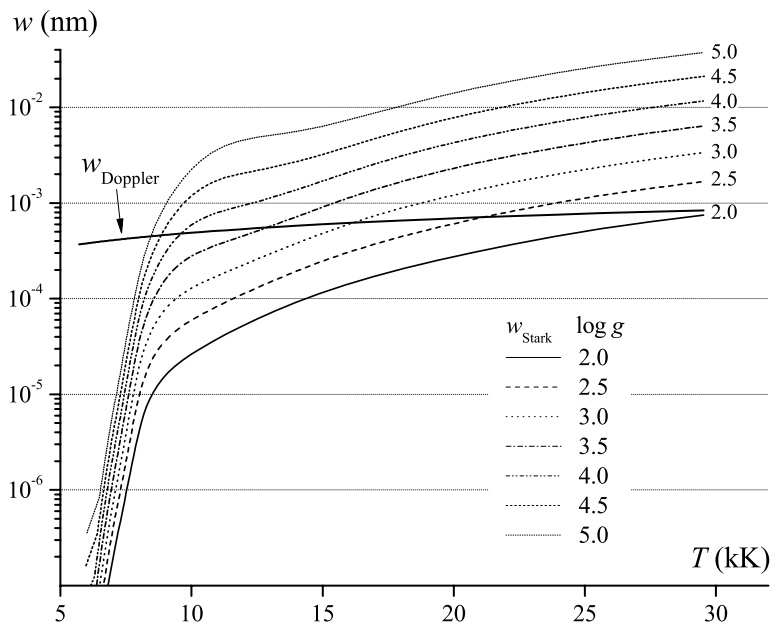

Fig. 2. Same as in Fig. 1 but Stark widths are shown for 7 values of model gravity $\log g=2-5, T_{\text {eff }}=10000 \mathrm{~K}$ and $v_{\mathrm{t}}=0 \mathrm{~km} \mathrm{~s}^{-1}$.

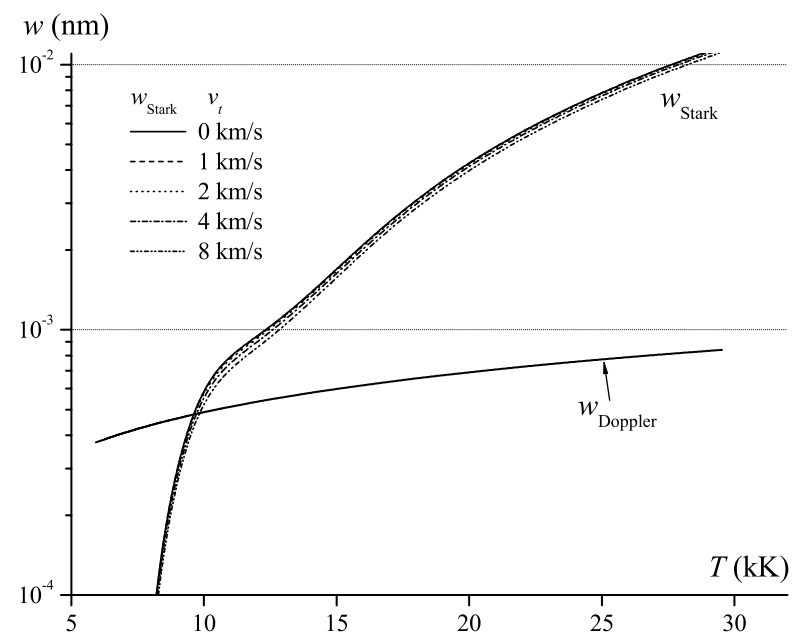

Fig. 3. Same as in Fig. 1 but Stark widths are shown for 5 values of turbulent velocity $v_{\mathrm{t}}=0-8 \mathrm{~km} \mathrm{~s}^{-1}, T_{\text {eff }}=10000 \mathrm{~K}$ and $\log g=4.0$.

and $v_{\mathrm{t}}=0 \mathrm{~km} \mathrm{~s}^{-1}$. The Stark broadening in stellar atmospheres with higher values of surface gravity is significantly larger than Doppler broadening. For stars with surface gravity $\log g=2$, Stark broadening is comparable to Doppler widths only for deeper hot atmospheric layers. For upper parts of stellar atmospheres $(T<10000 \mathrm{~K})$ Stark widths rapidly decrease and for layer temperature $T \approx 6000-7000 \mathrm{~K}$ Stark widths are several magnitudes lower than Doppler ones for all shown values of surface gravity $\log g$.

Figure 3 represents the dependence of Stark and Doppler widths on atmosphere layer temperature for a solar abundance Kurucz model $T_{\text {eff }}=10000 \mathrm{~K}, \log g=4$ and 5 , and values of the turbulent velocity $v_{\mathrm{t}}=0-8 \mathrm{~km} \mathrm{~s}^{-1}$. As one can see in Fig. 3, Stark widths do not show any significant dependence as a function of turbulent velocity.

The ratio of Stark and Doppler widths along the Hertzsprung-Russell diagram, from K0 to O5 spectral class, and within the range of the column mass at temperature minimum $\log m$ from -3 up to 0 are shown in Fig. 4 . In deeper parts of atmospheres, e.g. $\log m=0$, Stark broadening is larger than 


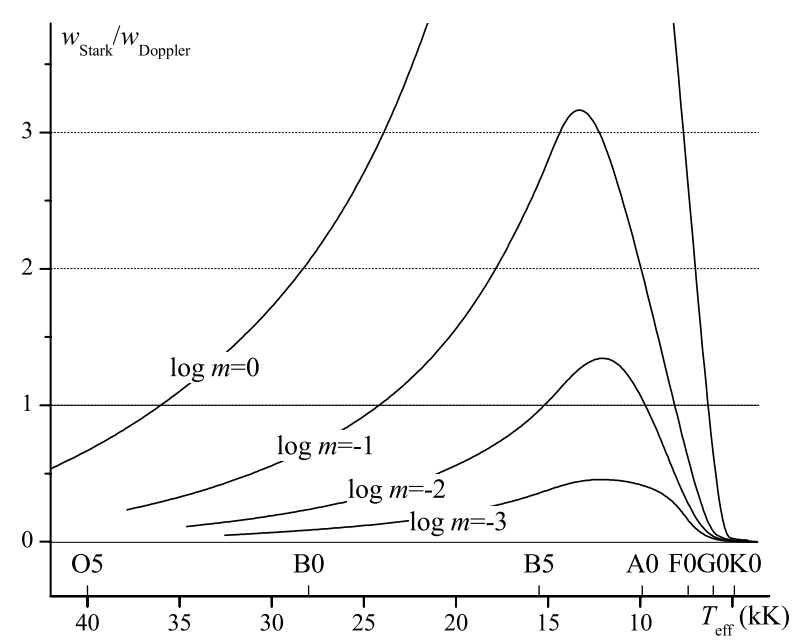

Fig. 4. Ratio of Stark and Doppler widths $w_{\text {Stark }} / w_{\text {Doppler }}$ as a function of the model effective temperature $T_{\text {eff }}$ (upper part of $x$-axis is spectral class). Dependance is shown for 4 values of logarithm of the column mass at temperature minimum $\log m$ from 0 to $-3, \log g=4.0$ and velocity $v_{\mathrm{t}}=0 \mathrm{~km} \mathrm{~s}^{-1}$.

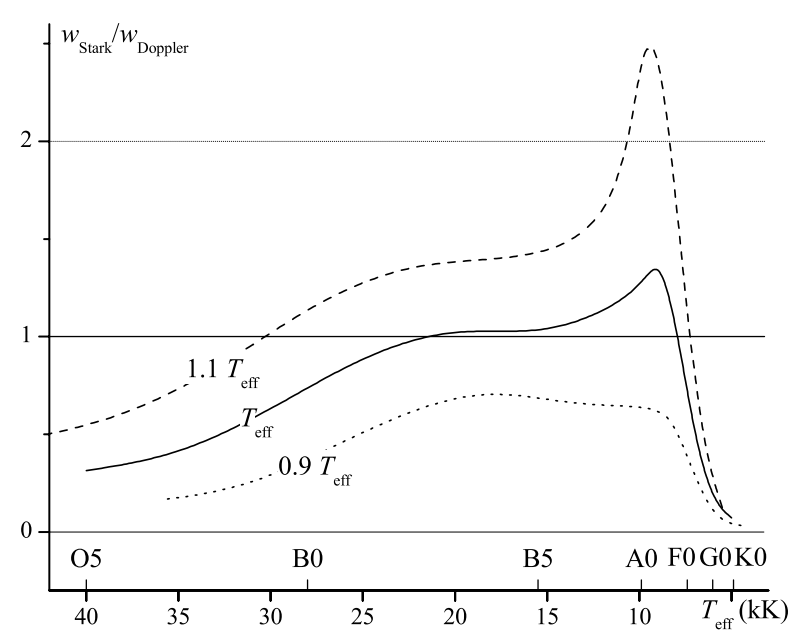

Fig. 5. Same as in Fig. 4 but the ratio is shown for 3 values of layer temperature $T=0.9 T_{\text {eff }}, T_{\text {eff }}$ and $1.1 T_{\text {eff }}, \log g=4.0$ and velocity $v_{\mathrm{t}}=0 \mathrm{~km} \mathrm{~s}^{-1}$.

the Doppler one for stars of G, F, A and B spectral type. For $\log m=-3$ (upper atmospheric parts) Doppler widths are comparable (approximately one-half) with Stark widths for spectral classes A0 to B5.

In Fig. 5 are shown ratios of Stark and Doppler widths as a function of star effective temperature (spectral class) for a Kurucz atmospheric model $\log g=4$ and $v_{\mathrm{t}}=0 \mathrm{~km} \mathrm{~s}^{-1}$. Stark and Doppler width ratio is given for 3 values of layer temperature $T=0.9 T_{\text {eff }}, T_{\text {eff }}$ and $1.1 T_{\text {eff }}$. In this figure one can see that Stark broadening is the dominant broadening mechanism for deeper atmospheric layers $\left(T=1.1 T_{\text {eff }}\right)$ for stars of $\mathrm{G}, \mathrm{F}$, A and B spectral class. For upper layers $\left(T=0.9 T_{\text {eff }}\right)$ Stark broadening is comparable to the Doppler one and should be taken into account for stars of A and B spectral class, since differences of Lorentz (for Stark) and Gauss (for Doppler) distributions may be important in the line wings.

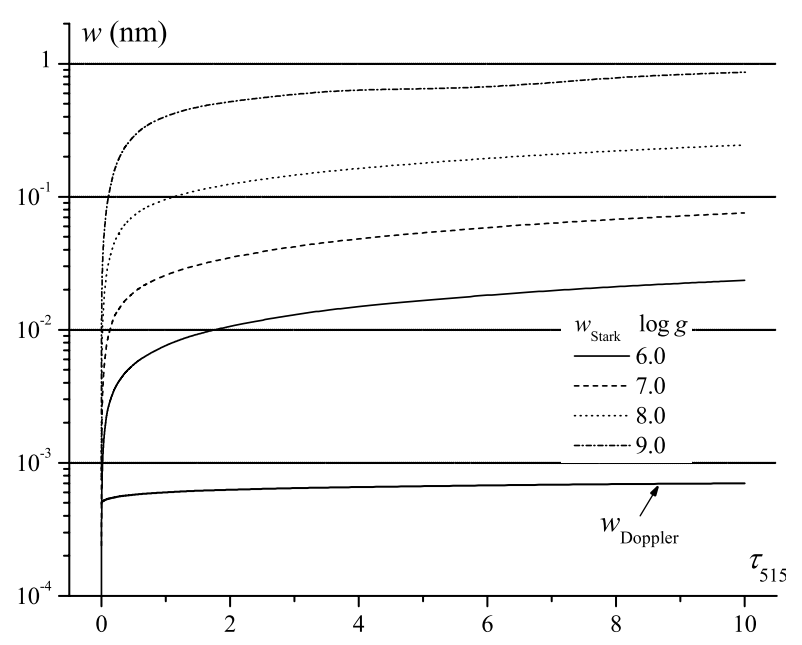

Fig. 6. Stark and Doppler widths for Cd III $\lambda=144.754 \mathrm{~nm}$ spectral line as a function of optical depth for standard wavelength $\lambda_{\mathrm{st}}=$ $515 \mathrm{~nm}$ for DA white dwarfs. Widths are given for 4 values of logarithm of surface gravity $\log g=6.0-9.0$. Effective model temperature is $T_{\text {eff }}=15000 \mathrm{~K}$.

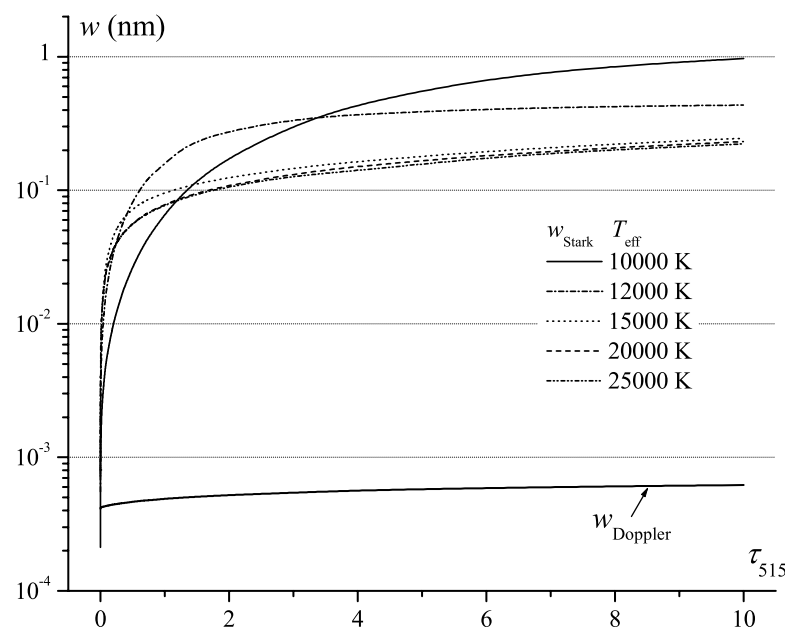

Fig. 7. Same as in Fig. 6 but widths are given for 5 values of effective temperature $T_{\text {eff }}=10000-25000$ and $\log g=8$.

To compare Stark and Doppler broadening we have calculated spectral line widths for Cd III $\lambda=144.754 \mathrm{~nm}$ for DA and DB white dwarf atmospheres. Models were taken from Wickramasinghe (1972). DA dwarfs are helium and metal underabundant and DB white dwarfs are helium and metal overabundant compared to hydrogen.

As one can see in Figs. 6-9 Stark broadening is by one or two order of magnitudes higher than Doppler one. Consequently, with the increases in pressure, electron density or effective temperature in DA and DB white dwarf models, the importance of Stark broadening increases as well.

The comparison of Stark and thermal Doppler contributions to the line widths is calculated for one line of $\mathrm{Cd}$ III $(\lambda=$ $144.754 \mathrm{~nm}$ ), and we can expect a similar contribution of the Stark broadening for all UV Cd III lines. The contribution of the Stark effect to line widths increases with the principal quantum number (Vince \& Dimitrijević 1985) and also one should take into account that in some cases, due to close perturbing 


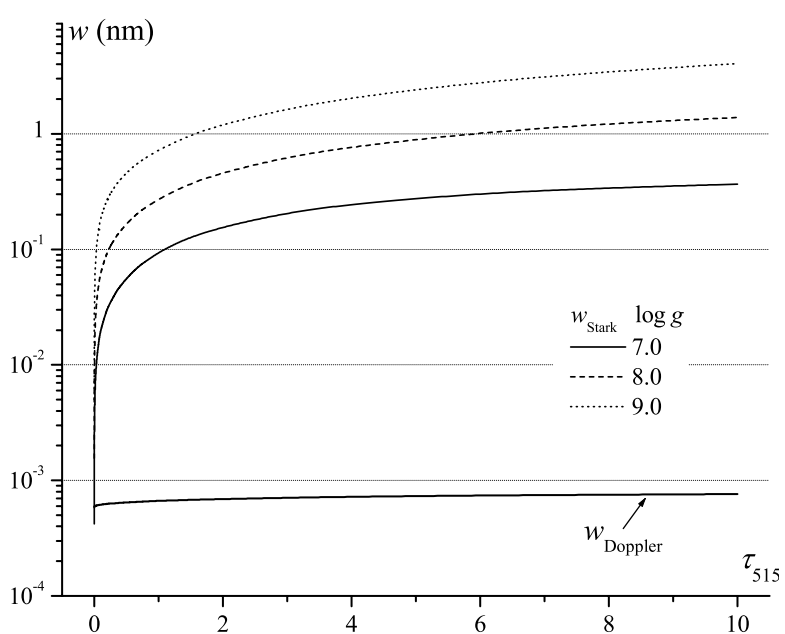

Fig. 8. Same as in Fig. 6 but for DB white dwarfs. Effective model temperature is $T_{\text {eff }}=20000 \mathrm{~K}$.

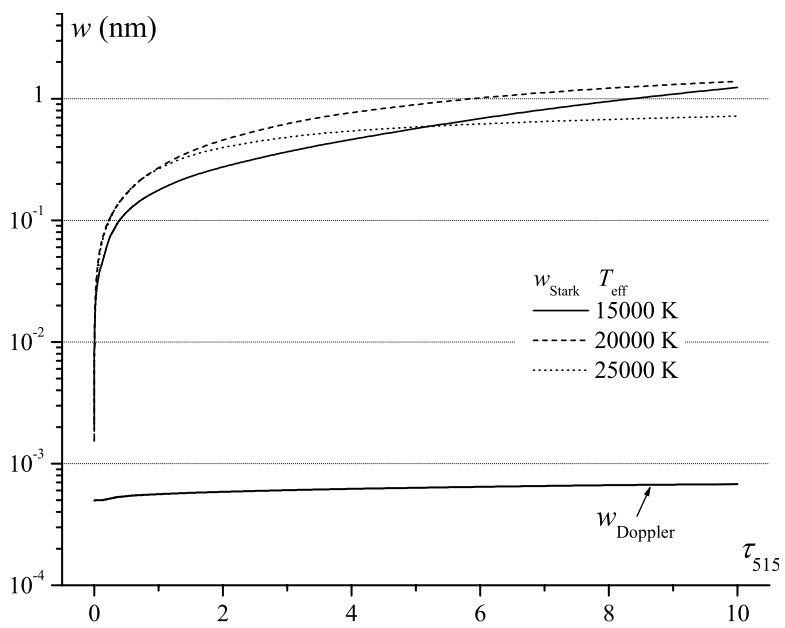

Fig. 9. Same as in Fig. 8 but widths are given for $T_{\text {eff }}=10000 \mathrm{~K}$, $20000 \mathrm{~K}$ and $25000 \mathrm{~K}$ and $\log g=8$.

levels, Stark widths might also be large. In some cases the Stark broadening may significantly contribute to the line widths as well as to the line shapes (see e.g. Dimitrijević et al. 2003, as well as results of our similar investigations for $\mathrm{Zr}$ II and $\mathrm{Zr}$ III in Popović et al. 2001a and Nd II in Popović et al. 2001b).

\section{Conclusion}

We have calculated Stark broadening parameters, widths and shifts, for $84 \mathrm{UV}$ spectral lines of Cd III by using the MSE method. We estimate that the errors bars of obtained data are $\pm 50 \%$. There is no possibility for the moment to calculate them in an adequate way by more sophisticated semi-classical methods since a sufficiently complete set of reliable experimental Cd III atomic energy levels is not available. When results of reliable Stark broadening calculations are not available (for example for Cd III lines not included here) more approximate methods are often used for stellar atmosphere analysis, e.g. data obtained by using methods based on regularities and systematic trends (see e.g. Lakićević 1983; Purić et al. 1991), on classical approaches, other approaches (see e.g. Griem 1974 and references therein) or even sometimes the value of 1 to 10 times the classical damping (see e.g. Lanz et al. 1988).

There are no measured or even theoretically calculated electron-impact broadening parameters for Cd III. Our analysis of the influence of Stark broadening on the Cd III $144.754 \mathrm{~nm}$ spectral line demonstrates the importance of this broadening mechanism for hot star atmosphere analysis. However, for very hot stars, of the spectral class $\mathrm{O}$, the influence of Stark broadening is reduced due to lower pressure in lower atmospheric layers. The effect of electron-impact broadening for DA and DB white dwarfs is even more significant.

Since with the development of space-born techniques, more good quality data on trace elements spectra will become available, we hope that the presented set of Cd III electron-impact broadening parameters will be of interest for future investigations of astrophysical and laboratory plasma, modelling of stellar atmospheres and stellar spectra synthesis and analysis. An example of the interest for as possible a large set of atomic data is the PHOENIX (see e.g. Hauschildt \& Baron 1999) computer code for stellar modelling which includes a database containing $4.2 \times 10^{7}$ atomic, ionic and molecular spectral lines. Moreover, data on Stark broadening of Cd III spectral lines might be of interest not only for modelling and interpretation of stellar spectra but also for abundance determination and investigation of stratification of chemical elements (see e.g. Dimitrijević et al. 2003).

Acknowledgements. This work is a part of the projects "Influence of collisional processes on astrophysical plasma lineshapes" (GA 1195) and "Astrophysical Spectroscopy of Extragalactic Objects" (GA 1196) supported by the Ministry of Science, Technologies and Development of Serbia.

\section{References}

Baranger, M. 1958a, Phys. Rev., 111, 481

Baranger, M. 1958b, Phys. Rev., 111, 494

Baranger, M. 1958c, Phys. Rev., 112, 855

Bates, D. R., \& Damgaard, A. 1949, Phil. Trans. Roy. Soc., A242, 101

Dimitrijević, M. S. 1997, Ap\&SS, 252, 415

Dimitrijević, M. S., \& Konjević, N. 1980, JQSRT, 24, 451

Dimitrijević, M. S., \& Kršljanin, V. 1986, A\&A, 165, 269

Dimitrijević, M. S., \& Sahal-Bréchot, S. 1984, JQSRT, 31, 301

Dimitrijević, M. S., Feautrier, N., \& Sahal-Bréchot, S. 1981, J. Phys., B14, 2559

Dimitrijević, M. S., Sahal-Bréchot, S., \& Bomier, V. 1991, A\&AS, 89, 581

Dimitrijević, M. S., Ryabchikova, T., Popović, L. Č., Shulyak, D., \& Tsymbal, V. 2003, A\&A, 404, 1099

Griem, H. R. 1974, Spectral Line Broadening by Plasmas (New York and London: Academic Press)

Hauschildt, P. H., \& Baron, E. 1999, J. Comput. Appl. Math, 109, 41

Kolb, A. C., \& Griem, H. R. 1958, Phys. Rev., 111, 514

Konjević, N. 1999, Phys. Rep., 316, 351

Konjević, N., \& Uzelac, N. J. 1990, JQSRT, 44, 61

Kurucz, R. L. 1979, A\&AS, 40, 1

Lakićević, I. S. 1983, A\&A, 127, 37

Lang, K. R., \& Wilson, R. F. 1978, MNRAS, 183, 5

Lanz, T., Dimitrijević, M. S., \& Artru, M.-C. 1988, A\&A, 192, 254 
Leckrone, D. S., Proffitt, C. R., Wahlgren, G. M., Johansson, S. G., \& Brage, T. 1999, AJ, 117, 1454

Oertel, G. K., \& Shomo, L. P. 1967, ApJS, 16, 175

Piskunov, N. E. 1992, in Stellar Magnetism, ed. Yu. V. Glagolevskij, \& I. I. Romanyuk (St. Petersburg: Nauka), 92

Popović, L. Č. 1994, Publ. Astron. Obs. Belgrade, 46, 1

Popović, L. Č., \& Dimitrijević, M. S. 1996, A\&AS, 120, 373

Popović, L. Č., Dimitrijević, M. S., \& Ryabchikova, T. 1999, A\&A, 127, 295

Popović, L. Č., Milovanović, N., \& Dimitrijević, M. S. 2001a, A\&A, 365,656

Popović, L. Č., Simić, S., Milovanović, N., \& Dimitrijević, M. S. 2001b, ApJS, 135, 109

Purić, J., Ćuk, M., Dimitrijević, M. S., \& Lesage, A. 1991, ApJ, 382, 353
Ralchenko, Yu. V., Griem, H. R., Bray, I., \& Fursa, D. V. 1999, Phys. Rev. A, 59, 1890

Sahal-Bréchot, S. 1969a, A\&A, 1, 91

Sahal-Bréchot, S. 1969b, A\&A, 2, 322

Seaton, M. J. 1988, J. Phys. B, 21, 3033

Shenstone, A. G., \& Pittenger, J. T. 1949, J. Opt. Soc. Am., 39, 219

Shore, B. W., \& Menzel, D. H. 1965, ApJS, 12, 187

Van Kleef, Th. A. M., Joshi, Y. N., \& Uijlings, P. 1980, Phys. Scr, 22, 353

Vince, I., \& Dimitrijević, M. S. 1985, Publ. Obs. Astron. de Belgrade, 33,15

Wickramasinghe, D. T. 1972, Mem. R. Astron. Soc., 76, 129

Zeng-xin, Z., Jian-min, Y., \& Yong-sheng, S. 1999, Chin. Phys. Lett., 16,885 